\title{
Taking a Drive, Hitching a Ride: Autonomous Driving and Car Usage
}

\author{
Eva Fraedrich and Barbara Lenz
}

\subsection{Introduction}

For over a century, the automobile has shaped our physical mobility like no other means of transport. For almost as long, however, it has also been the subject of criticism surrounding the ecological, social and health consequences of automobility.

Autonomous driving may bring with it upheaval in how individuals and society deal with the car, and thus also impact transport, mobility or urban structures. Recently, it has received much public attention - in the mass media it has become part of regular news reporting and is even taken up, discussed and shared on social platforms. There is public discussion of how technology is currently (perceived) to be developing, but also of the possible radical change in how cars will be used, which would be accompanied by altered attitudes to cars and driving. Expected changes, for instance, include a dramatic drop in the current rate of car ownership [1,2], a reduction in the space currently needed for parking [3], and a transformation in car ownership and usage in favor of carsharing fleets [4].

The topic's growing prominence demonstrates that the debate surrounding autonomous driving and autonomous vehicles is evidently cropping up more and more in society. In any transition to a potential new era of car ownership and usage, it is important to ask early on what people associate with autonomous driving. How do they perceive the technology and the ensuing debate? In what contexts is such a debate embedded? What

E. Fraedrich $(\square) \cdot$ B. Lenz

Geography Department, Humboldt-Universität zu Berlin, Berlin 10099, Germany

e-mail: eva.fraedrich@geo.hu-berlin.de

B. Lenz

e-mail: barbara.lenz@dlr.de

B. Lenz

German Aerospace Center (DLR), Institute of Transport Research, Berlin 12489, Germany 
are people's hopes, fears, and fantasies? Where is the technology viewed with optimism or skepticism, as being feasible or even as impossible?

An initial systematic in-depth structuring of the topic from road users' point of view has focused on the wealth of various topics, perceptions, and assessments presently associated with autonomous driving (for details of this exploratory study's survey and findings see Chap. 29). In this connection, it has become clear that autonomous driving is assessed strongly from a subjective usage context, and is also tightly bound up with the motives, value systems, and practices of using private cars. The choice of transport mode - the decision to use a particular transport means - is in turn a deeply rooted part of our everyday praxis, which can be expected to change little by little rather than by leaps and bounds (cf. [5, 6]). It is not currently clear what a future with autonomous vehicles might look like, and which areas of our daily lives, communal life, or mobility will be subject to change. Examining autonomous driving within the scope of everyday practices, of which car usage and ownership form part makes the context of the technology more conceivable.

This article first looks at the latest research on car use and ownership. Using selected findings from previous surveys, we then direct the focus to use-related perspectives on autonomous driving (these perspectives specifically refer to the use cases developed within the Villa Ladenburg project see Chap. 2). In this context, we will also examine the role of motivations for using and owning cars.

\subsection{We Drive ... and Drive ... and Drive ...}

The question of why people use cars has occupied scientists of various disciplinesincluding psychology, sociology, transportation science, economics and social sciences in particular - for almost as long as the automobile has been in existence [7]. This debate has been a highly emotional one again and again (cf. [7, 8]); literature and studies on the individual and societal benefits of car usage are clearly in the minority (see $[9,10]$ ). In light of the discussion of more sustainable ways of living, to which reducing motorized personal transport could significantly contribute, the search for the reasons behind car usage has intensified in recent years, which can be seen in the increasing numbers of both academic and popular-science papers and articles on the subject.

The automobile is part of a complex, non-linear system - the system of automobilitywhich always reproduces the preconditions for its own expansion (which can be seen in potent and complex technical, political and social relationships between industry, suppliers, infrastructure, resource use, and urban and regional planning etc. [11]). This has led to the onset of path dependency and a "lock-in" effect which can hardly be reversed (see [11-13]). Stotz (2001) points out how the car as a technical artifact is actively integrated into social and societal (socialization) processes, and in this way acquires the status of subject rather than of an object (see [14]). Other authors have also remarked that the car itself has not appeared to be an external object for quite some time now, but rather seems to be a symbolic extension of the human body (see [15-17]). There are also a series of 
studies, however, that highlight the car's significance for physical and mental well-being, and even for higher life expectancy (see [18-20]).

The motivations that have been empirically uncovered for using or owning a car are manifold. In the following, we shall briefly outline the foremost and most up-to-date attempts at explanation.

\subsubsection{What Are the Reasons and Motives for Using Cars?}

\section{Instrumental motives}

For a long time, the motives for using and owning cars was explained by behavioral models mainly based on instrumental or utilitarian aspects (see [21]). In order to fulfill their everyday mobility needs - that is to say, to undertake various activities at various locations - people resort to several means of getting around (car, bike, train, etc.). The reasons for this, goes the assumption, are based on the specific benefits they bring with them, e.g. availability, speed, monetary costs, flexibility, safety, convenience, etc. Whoever, therefore, chooses the car to get around-when, for example, desiring to get from A to B more quickly, or setting off on holiday in as good-value and comfortable a way as possible, or getting to work when it is far from home-deems it more favorable than other transport means. Whether the advantages a car brings are objectively measurable is a secondary matter here; what is at issue is a subjectively perceived benefit, which need not correspond to any actual one (see [22]).

Urban development concepts such as "New Urbanism" or "Smart Growth" primarily involve the assumption that instrumental aspects are decisive in how transport modes are chosen. This then shapes infrastructural, transport-planning, and policy activities - areas with greater settlement density and mix of uses (residential, work, shopping, leisure) aim to shorten trip lengths and times, and should enable a reduction in motorized private transport (see [23-26]).

\section{Affective motives}

Instrumental reasons alone, however, are not sufficient in themselves to fully explain decisions made when choosing transport modes [27, 28]. Furthermore, they cannot always be clearly distinguished from other motives. Independence and freedom, the dominant reasons for using cars, can both be interpreted as instrumental and affective aspects. The freedom - free from timetables, for example - to drive off at any moment can be a rational basis for taking the car. At the same time, this freedom can also give a feeling of autonomy and independence — an emotionally colored reason to drive (see [6, 29]).

Affective or emotional factors play a significant role in both everyday driving and journeys made for leisure. Transport means such as the car are tightly bound up with emotions and feelings that in turn exert considerable influence on what modes are, and are 
not, selected. This is the case even though car users are often inclined, in direct surveys, to rationalize their motives and motivations, and let emotional aspects 'go by the board' [28]. Driving can thus be accompanied by feelings of relaxation and pleasure, excitement and elation, the joy of driving or of speed, but also by stress and tension. As a rule, car driving is associated with positive feelings [30-32].

\section{The car as status symbol, symbolic motives and cultural symbolic meaning}

Alongside instrumental and affective motives, symbolic motives also influence the choosing of cars (or transport modes in general). In doing so, they fulfill two functions: First, a personal value may be expressed by using or owning a specific vehicle. Second, this may then underscore a certain position in the social order. A car may, for instance, signify status and prestige, or perhaps a particular attitude to life [28-30, 33, 34].

Furthermore, cars also serve as a cultural symbol. They represent progress, freedom, individuality, and sophistication. They inspire music, art, literature, film, and advertising. They have an impact on family life, social interaction, and cultural rituals, and are an integral component of initiation rites in modern society (see [11, 35-37]).

\section{Further approaches}

Beyond the not always clear-cut distinctions of instrumental, affective, and symbolic motives (for more on this, see [6, 38]), a number of recent studies and debates have placed cars and the reasons for using them more clearly in the context of a sociotechnical system. This research has thrown light on the way cars fulfill specific social functions: how, for instance, they perpetuate geographical inequalities and divisions, shape social constructions, or how they can fix national and cultural identities [14, 39-43].

A further important topic, in the context of our work on the interrelationship between car ownership and autonomous driving, is the car as "private space" (see [44, 45]). For some, the "cocoon" car, an "inhabited" space, can function as a refuge from what is felt to be stressful, hectic, loud, and overcrowded modern life. Moreover, it also holds special significance for social interaction. Laurier und Dant [46] have examined the significance of this "inhabited" space and the emergent increasing vehicle automation. They point out that liberation from the driving task fits in with cars' evolutionary development over recent decades. This trend is far less a matter of expressing identity (as also manifested in sports cars' loss of significance and the increase in closed vehicles with large interiors) than one of temporarily "inhabiting" a space that also enables social interaction. Such studies consistently stress that it is social factors, far more than individual ones, that can play a considerable role in the way cars are favored. Inside an automobile, people take on the same specific social roles that they assume in their other social interactions (being a parent, friend or worker, etc.) [42, 47].

In this context, newer studies on how people value their time spent travelling when using various transport modes could also be of use (see [27, 48]). For a long time, driving cars was viewed as unproductive, lost time - the frequently monotonous, unvarying way 
to work was a typical example of this burden [49]. That time spent in a car is certainly subjectively perceived as valuable, however, and may even be viewed as a "gift," has been recently demonstrated by Jain and Lyons [48] with their study on commuters. Travel times were here seen as time for relaxing from everyday hustle and bustle, a transition time between work and home. These findings are in line with an earlier work on commuting, in which Mokhtarian et al. (2001) were already able to show that time spent in a car is certainly not always seen as wasted [27].

\subsubsection{Summary}

At present, car usage and ownership is approaching a saturation point in industrial nations, which is not to say that the car will fall from its dominant position so easily because of this [50]. For the vast majority of car users, it will far more likely retain its significance in fulfilling their daily needs ([51]: 114). What is of prime importance in the debate on the future uses of autonomous cars (and also their acceptance) is to take into consideration the complexity of instrumental, affective, and symbolic aspects. Technology's perceived functional qualities can, at first glance, appear to be an easily measurable "objective" benefit, but these functional aspects only reveal their significance in the context of the subjective motivations — affective and symbolic - that accompany autonomous driving. Furthermore, embedding instrumental motives for (autonomous) car usage in the context of everyday praxis, and also of the relevant sociotechnical system, plays a role too. The autonomous vehicle may be perceived as safe, flexible, and comfortable, but this will only lead to it actually being used if safety, flexibility or comfort have a specific significance in daily praxis [51].

\subsection{Multiple-Method Investigative Approaches for Autonomous Driving in the Context of Car Usage and Ownership}

The insights from research into car usage and ownership, together with the indications from the first exploratory study on autonomous driving, have formed the conceptual basis for our follow-up empirical work. The objective of these ongoing studies is to survey, in a use-oriented fashion, the specific attributes that road users currently ascribe to autonomous cars and, in the "translation" of such attributes, to decipher instrumental, affective and symbolic motives. The questions guiding the research are thus: What characteristics and assessments do road users associate with autonomous driving and autonomous vehicles? What various motives are decisive in this? The context of what attributes are currently ascribed to autonomous driving is also to be analyzed and, where possible, linked to how people live their daily lives and get around. In this way, it will then be possible to determine which attitudes and judgments may be expected to have an effect on acceptance of the technology. 
To this end, a multiple-method procedure was selected. To explore potential variations in people's perceptions and judgments, respondents were confronted with specific use cases of autonomous driving in a quantitative online survey. This survey also contained a free-text questionnaire-participants were thus able to ascribe autonomous driving with attributes in a completely individual way. A more in-depth analysis encompassing the context in which autonomous driving is presently perceived and viewed allowed for exploratory qualitative procedures to be pursued in parallel as part of group discussions. The focus here lay particularly on ambivalence in how technology is viewed (see Chap. 29), particularly in advance of potential implementation — with both fear and high expectations. Exploratory qualitative procedures appear to be especially apt for this still relatively young field of research. Until now, there has been barely any insight available into the attitudes and motives surrounding autonomous driving on the user side - the open character of such studies helps to decipher these motivations.

\subsubsection{Perception and Assessment of Autonomous Driving in Relation to Specific Use Cases}

It may also be expected that each of the four use cases developed in the course of this project (Chap. 2) will be perceived and assessed differently in view of their acceptance-relevant aspects. This is because they not only differ in technical terms, but also in the specific implications, usage areas, and attributes they are associated with (for more on this see Chaps. 6, 11, 12, and 32). In a quasi-representative survey, we therefore recorded differentiated attitudes to autonomous driving with recourse to the use cases. A thousand people were surveyed, and represented the total German population regarding gender, age, income, and level of education. An in-depth discussion of the survey-which was developed and carried out in cooperation with several authors of the present volume - and sampling can be found in Chap. 6.

A total of $57 \%$ of those surveyed declared they were generally interested in the topic of autonomous driving. However, $44 \%$ stated they had no knowledge of the subject, and a mere $4 \%$ termed themselves as being well informed or having specialist knowledge at all, let alone being an expert. $78 \%$ obtain their information on the topic primarily from the mass media, $64 \%$ go straight to experts, $56 \%$ discuss it with friends or co-workers, and $40 \%$ share and compare notes on social media.

Following a general section with questions on sociodemographics, mobility behavior, travel requirements, etc. the respondents were each randomly allocated one of four scenarios based on the use cases. Each scenario was introduced with a short description:

Scenario for Interstate Pilot Using Driver for Extended Availability: On freeways or similar roadways, the driver can hand over control to the vehicle. The driver does not have to pay attention to other traffic or the driving task in this time, and can pursue other activities.

Scenario for Autonomous Valet Parking: After all passengers have got out of the vehicle, it can drive itself to a pre-determined parking space and also from there to a pick-up address. 
Scenario for Full Automation Using Driver for Extended Availability: Whenever and wherever desired or required, the driver can hand over control to the vehicle. The driver does not have to pay attention to other traffic or the driving task in this time, and can pursue other activities.

Scenario for Vehicle on Demand: A vehicle on demand drives its passengers without the presence of any human driver. People themselves can no longer drive in such vehicles - the vehicle's interior will thus also have neither steering wheel nor pedals.

A follow-up question to each use case as it was introduced enquired whether the respondents would essentially be prepared to replace their till-now preferred transport mode with an autonomous vehicle. This question has had already been put to them in the same form in the first, general part of the survey, but there it was relatively unspecifically termed "an autonomous vehicle" with no further explanation. Altogether the respondents had relatively little desire to replace their own vehicle (or "favorite mode of transport") with an autonomous vehicle-whether precisely specified or not. Only between 11 and $15 \%$ agreed to this statement in large or full measure (see Fig. 31.1). However, $27 \%$ said they could hardly imagine, or could not imagine at all, replacing their preferred mode with a (non-specified) autonomous vehicle. When, as in the survey, autonomous driving is proposed in relation to a specific use case, this degree of refusal increases sharply, to between 44 and $54 \%$. This means that refusal becomes stronger with more precise scenario descriptions. The lowest acceptance, incidentally, is for Vehicle on Demand$54 \%$ would not wish to replace their favored mode with it and only $11 \%$ could envisage it at all.

To explore what the respondents currently associate with autonomous vehicles, they were asked to declare in their own words, in up to fifteen free-text boxes, what they understand by the term "autonomous vehicle." The short descriptions (see above) were also a basis here. The following analysis refers solely to the answers of those respondents

\section{In principle, I can imagine replacing my favorite mode of transport with an autonomous vehicle.}

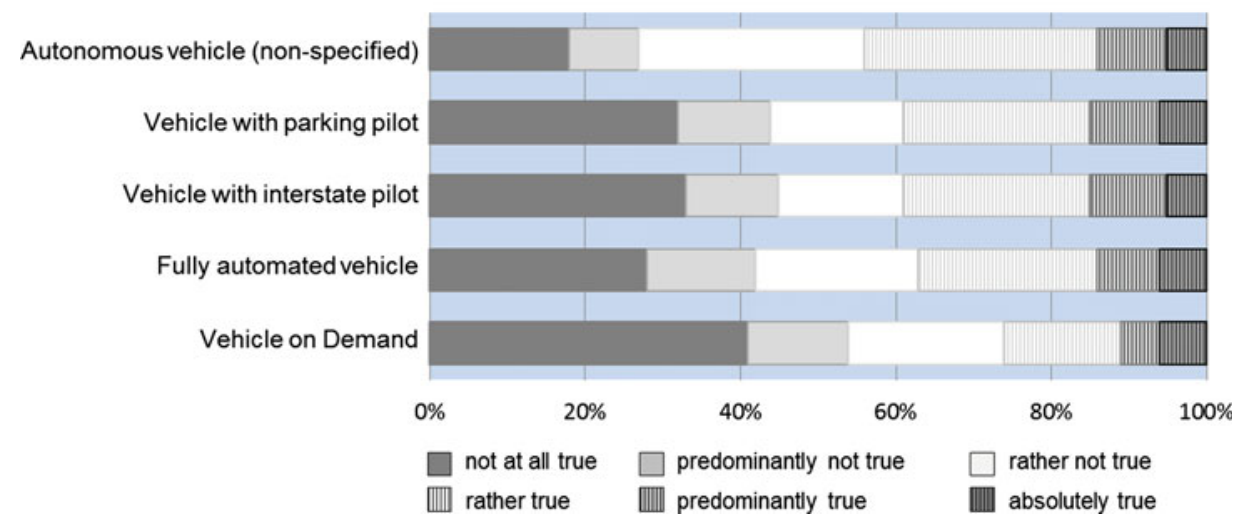

Fig. 31.1 Preparedness to replace the preferred means of transport with an autonomous vehicle 
who had been allocated the "Full Automation Using Driver for Extended Availability" (referred to as "Fully automated vehicle" in the following) and "Vehicle on Demand" use cases.

The answers of the 250 respondents were summarized and categorized by hand, then allotted specific connotations (see below). For "Fully automated vehicle" there were a total of 3750 entries; of these, 2587 (69 \%) were invalid for various reasons because it was not possible to make out a reference to the question, for example. For Vehicle on Demand, there were also 3750 entries overall, of which 2512 (67\%) were unusable. Figure 31.2 shows the distribution of statements with various connotations: positive, ambivalent, negative or without connotation - the invalid entries have already been taken out at this point, the percentages refer to the remaining mentions.

While the majority of descriptions of Fully automated vehicle are positive, the same can only be said of $38 \%$ of definitions applied to Vehicle on Demand. For these two use cases, 36 and $40 \%$ of the statements respectively had negative connotations. A small portion of the perceptions ( 5 and $4 \%$ respectively) were ambivalent, i.e. they could not clearly be connoted as positive or negative.

Overall, the qualities that the respondents attributed, independently of one another, to each of the two scenarios turned out to be relatively similar. Many answer categories are both equivalent in meaning and similar in their percentage distribution. We shall turn next

Fig. 31.2 Connotations regarding autonomous driving: fully automated vehicle and vehicle on
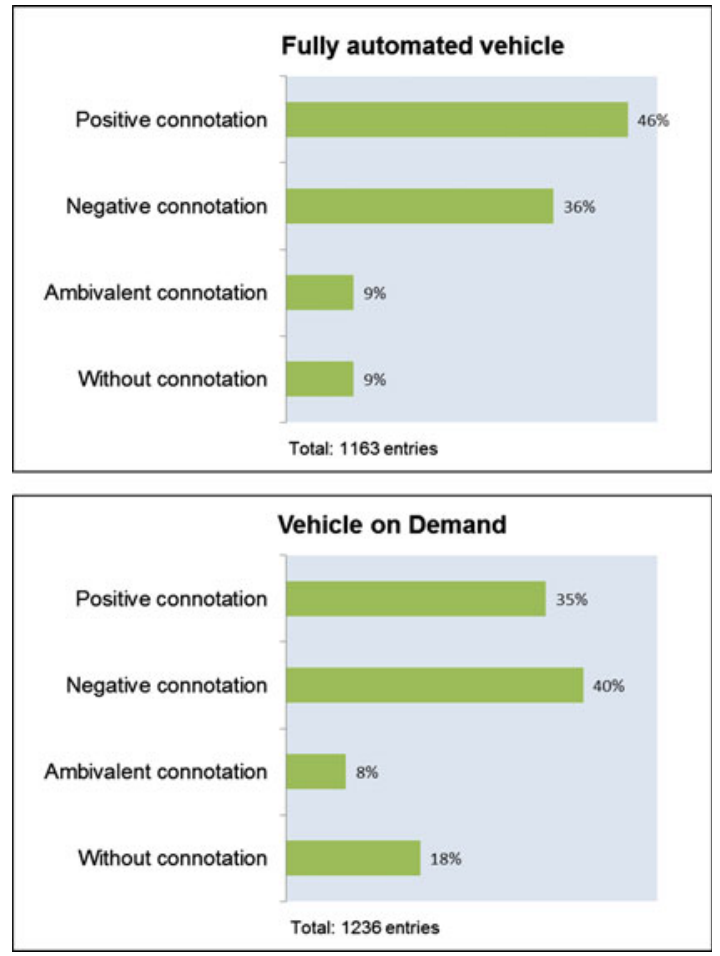
to some of the notable differences. We shall also examine the attributes given, where possible, for what instrumental, emotional, and symbolic characteristics are ascribed to autonomous vehicles.

\section{Full Automation Using Driver for Extended Availability}

In the "positive assessments" segment, $17 \%$ of statements were in the category of "comfortable," followed by "good" (13\%), "safe" (11\%), "relaxing" (10\%), and "modern" (10\%)-these proportions are given in Table 31.1. Among the "ambivalent assessments," $8 \%$ of the answers came in the "luxury" category, and $15 \%$ of all

Table 31.1 Answers in the description field "Fully automated vehicleis..."-summarized and categorized

\begin{tabular}{|c|c|c|c|c|c|}
\hline Positive & in $\%$ & Ambivalent & in $\%$ & Negative & in $\%$ \\
\hline Comfortable & 17 & The future & 48 & Not for me & 16 \\
\hline Good & 13 & Utopian & 23 & Expensive & 15 \\
\hline Safe & 11 & Needs getting used to & 22 & Unnecessary & 12 \\
\hline Relaxing & 10 & Luxury & 8 & Weird & 11 \\
\hline Modern & 10 & Total: 106 & 100 & Unsafe & 11 \\
\hline Practical & 9 & & & $\begin{array}{l}\text { Insufficiently } \\
\text { developed }\end{array}$ & 9 \\
\hline Brilliant & 7 & No valuation & in $\%$ & Boring & 7 \\
\hline Efficient & 6 & Autonomous & 29 & $\begin{array}{l}\text { Technology } \\
\text { dependent }\end{array}$ & 7 \\
\hline Interesting & 5 & No idea & 20 & Unpredictable & 7 \\
\hline $\begin{array}{l}\text { For the mobility- } \\
\text { impaired }\end{array}$ & 4 & $\begin{array}{l}\text { Incomprehensible } \\
\text { statement }\end{array}$ & 18 & Dangerous & 3 \\
\hline Helpful & 3 & $\begin{array}{l}\text { Similar to other } \\
\text { transport modes }\end{array}$ & 12 & Terrible & 1 \\
\hline Exciting & 2 & Not vehicles & 11 & Total: 422 & 100 \\
\hline $\begin{array}{l}\text { Environmentally } \\
\text { friendly }\end{array}$ & 2 & A car & 6 & & \\
\hline Flexible & 2 & $\begin{array}{l}\text { Comprehension } \\
\text { questions }\end{array}$ & 5 & & \\
\hline Total: 533 & 100 & Total: 102 & 100 & & \\
\hline
\end{tabular}


"negative assessments" were because they viewed the scenarios as "expensive." In this negative segment, only the answer category of "not for me" was more highly represented, at $16 \%$. "Luxury" was not once mentioned in connection with Vehicle on Demand, however, and, at $7 \%$ of all statements, "expensive" only came in at seventh place.

In the positive assessments segment, Fully automated vehicle was mainly associated with functional or instrumental aspects - vehicles such as these are described as "comfortable," "safe," "practical," "efficient," "for the mobility-impaired," "helpful," "environmentally friendly," and "flexible." Vehicle on Demand was also deemed "useful," although no-one deemed it "helpful." Regarding Fully automated vehicle, only the positive attributes of "relaxing," "brilliant," and "exciting" could be termed as emotional or affective. The same applies for Vehicle on Demand, though the relatively weaker connotations of "great" and "good" categories were expressed instead of "brilliant." As for the negative assessments, on the other hand, the distribution of functionally versus emotionally connoted aspects is reversed. "Weird," "boring," "dangerous," and "terrible" are clearly affective categories (and are joined in the case of Vehicle on Demand by "scary"), while "expensive" and "insufficiently developed" denote more functional aspects. Answer categories that may be assumed to have a symbolic connotation are scarcely to be found among the statements. Statements in the "modern," "interesting" or "luxury" categories most likely indicate that such autonomous vehicles are perceived and assessed in terms of aspects concerning the status manifested in the vehicles themselves.

\section{Vehicle on Demand}

The summarized and categorized attributes given for the Vehicle on Demand can be seen in percentage in Table 31.2. The top answer categories in the area of "positive assessments" hardly differ from those for Fully automated vehicle. Only in first place do we see a completely new category of "useful," with $15 \%$ of all statements made in this segment. After this, the respondents go on to describe the vehicle as "comfortable" (14\%), "relaxing" (13\%), "modern" (12\%), and "safe" (10\%). Eighteen percent of statements made about Vehicle on Demand have no judgment attached to them. Half of these come under the "no idea" category — only $20 \%$ of respondents, in comparison, declared they had "no idea" about Fully automated vehicle. Only $2 \%$ of statements in the "no valuation" column were in the category of "similar to other transport modes," whereas this applied to $12 \%$ of those made about Fully automated vehicle. This category included entries such as "Fully automated vehicle is "like the railroad" or "Vehicle on Demand is "a taxi.",

A quarter of all negative statements on Vehicle on Demand fall under the highly emotionally cast categories of "scary" (10\%), "dangerous" (7\%), "weird" (6\%), and "terrible" (2\%). In contrast, not a single respondent found Fully automated vehicle to be "scary," and only $15 \%$ termed it "weird" (11\%), "dangerous" (11\%), or "terrible" (1\%). 


\subsubsection{Summary}

Overall the survey clearly demonstrates that Vehicle on Demand is the subject of the greatest number of negative and fewest positive assessments. Of the 250 respondents allocated to this use case, $54 \%$ could not imagine replacing their currently preferred mode of transport with a Vehicle on Demand. In direct comparison with Full Automation Using Driver for Extended Availability, Vehicle on Demand is described with rather negative statements, with a quarter of all descriptions even viewing it as scary, dangerous, weird, or terrible. Clearly, this means that fewer respondents could imagine using a Vehicle on Demand than one they can still drive themselves, as is shown by the number of statements professing "no idea."

Table 31.2 Answers in the description field "A Vehicle on Demand is..."-summarized and categorized

\begin{tabular}{|c|c|c|c|c|c|}
\hline & & & & \\
\hline Positive & in $\%$ & Ambivalent & in $\%$ & Negative & in $\%$ \\
\hline Useful & 15 & The future & 41 & Not for me & 16 \\
\hline Comfortable & 14 & Utopian & 41 & $\begin{array}{l}\text { Technology } \\
\text { dependent }\end{array}$ & 12 \\
\hline Relaxing & 13 & Needs getting used to & 18 & Unnecessary & 11 \\
\hline Modern & 12 & Total: 96 & 100 & Scary & 10 \\
\hline Safe & 10 & & & Unsafe & 10 \\
\hline Great & 9 & No valuation & in $\%$ & Unpredictable & 7 \\
\hline Interesting & 6 & No idea & 50 & Expensive & 7 \\
\hline Efficient & 5 & Autonomous & 22 & Dangerous & 7 \\
\hline $\begin{array}{l}\text { For the mobility- } \\
\text { impaired }\end{array}$ & 4 & $\begin{array}{l}\text { Incomprehensible } \\
\text { statement }\end{array}$ & 17 & $\begin{array}{l}\text { Insufficiently } \\
\text { developed }\end{array}$ & 6 \\
\hline Good & 4 & Not vehicles & 6 & Weird & 6 \\
\hline $\begin{array}{l}\text { Environmentally } \\
\text { friendly }\end{array}$ & 4 & A car & 2 & Boring & 5 \\
\hline Good value & 2 & $\begin{array}{l}\text { Similar to other transport } \\
\text { modes }\end{array}$ & 2 & Terrible & 2 \\
\hline Exciting & 2 & $\begin{array}{l}\text { Comprehension } \\
\text { questions }\end{array}$ & 1 & Total: 493 & 100 \\
\hline Total: 431 & 100 & Total: 218 & 100 & & \\
\hline
\end{tabular}


The answer categories of "expensive" and "luxury," which are either especially or exclusively attributed to Full Automation, indicate that such vehicles are still clearly linked with individual private ownership. Vehicle on Demand, on the other hand, is compared with other transport modes little or not at all.

Classifying the statements on autonomous driving in terms of their instrumental, affective or symbolic aspects revealed that instrumental attributes predominate among positively connoted assessments. Strongly emotional statements, on the other hand, form the majority of negative accounts. Descriptions foregrounding the status character of autonomous vehicles, in contrast, were scarcely to be found. In the qualitative survey described in the following section, we shall take a closer look at the perceived negative aspects of autonomous driving, and ask which sociodemographic contexts they are embedded in. Such hostile attributes, in particular, are evidently strongly aligned with subject-related and affective issues as well as the context of car use and ownership, as the exploratory study has already shown (Chap. 29).

\subsubsection{Autonomous Driving in the Future: "Do We Really Want to Live Like That?"'}

The following findings are based on three group discussions in Berlin whose composition varied each time. All participants had a high level of education; some were in academia, studying or working at a university or other research institutes. The discussions involved five, six, and seven participants respectively. All those involved were living in Berlin, seven of them were women. The gap between oldest and youngest was greatest in the first discussion round, with the youngest 20 years of age, the oldest 50. All participants regularly used a car, though not all of them owned one. At the end of each discussion, data on sociodemographics, transport behavior, and car use and ownership were collected. All those taking part had already heard of autonomous driving before the discussion sessions.

At the beginning of the session, the participants were given an illustrated narrative scenario of autonomous driving in the form of an A4 flyer. There were two different scenarios — one on "Full Automation Using Driver for Extended Availability," the other on "Vehicle on Demand." Each group was introduced to one of the scenarios, which they then considered within group-based introspection. The aim of this introspection is to make explicit the processes of "inward observation", that is to say, to consciously direct concentration and attention towards interior processes ([52]: 493, translation by the authors). Under the guidance of a research person, a group's participants address the research object in question and document their own inner processes and experiences independently of one another. Finally, they share what they have found via self-observation with the group without commenting or passing judgment on each other's accounts. In a second stage, the

\footnotetext{
${ }^{1}$ This question stems from a comment in the discussions described in this section.
} 
group members - keen explore the topic further from hearing others' experiences - add to their reports. Together with the scenario, the participants received the following instructions:

You have just received a short description of a scenario involving the cars of the future - a short story on what the driving of tomorrow might look like. Please read the story carefully. Try to imagine yourself in what you read and think about your feelings, fantasies, and sensations - be open for anything and everything that crops up as you think about the topic!

Please make notes on where your thoughts lead you.

The participants then each read out their notes in turn, as a so-called "introspection report." The reports are later transcribed and evaluated by means of qualitative heuristics. Unlike everyday procedures of discovery, which are frequently made unconsciously, qualitative heuristics is "rule-directed and supplied with a methodology," and takes the form of systematized and intersubjectively traceable search-and-find procedures ([53]: 226, translation by the authors). The method is based on four rules:

1. Openness of the research person and subjects

2. Openness of the research object

3. Maximum structural variation of perspectives

4. Analysis of commonalities.

Furthermore, qualitative heuristics uses the so-called dialogic principle: A question is asked of an object (in the study, the transcribed introspection reports) which gives "answers." New questions are then asked from another perspective, or a different angle, and so the process continues. The research object and research person are thus in close dialogic contact, which also serves to soften the strict division between (research) subject and (research) object.

After producing introspection reports, the groups started open discussions, which were aligned on the implicit behavioral patterns (see [54]) in car usage and ownership. In now giving our results, however, we shall focus on what the participants conveyed in their introspection reports.

\subsubsection{Results}

The scenarios allocated to the group discussion participants stimulated great interest in the topic, but also critical questioning. In what follows, we shall describe some examples of topics that had already proved significant in the earlier, exploratory survey. The introspection reports show an ambivalent attitude to autonomous driving that is comparable to the results of the study given in Chap. 29.

In addition, however, it became clearer which specific fears and worries relate to autonomous driving, and what social context it is seen in. We shall take a closer look at the range of hostility towards autonomous driving below - the results stem from both groups who were given the "Full Automation Using Driver for Extended Availability" scenario. 


\subsubsection{Skepticism About a Future with Autonomous Vehicles}

For the sake of completeness, it should be mentioned at this point that, in the scenario stories provided to the participants, the protagonist "Yvonne" used the time made free to her from not having to drive herself to pursue work activities (e.g. email correspondence), among other things. Although other activities were also covered alongside this, the story did tend to focus more on typical organizational activities (taking the kids to school, doing the shopping, etc.) than on leisure and relaxation pursuits (looking out the window, watching movies, sleeping/relaxing, etc.). It is therefore possible that skeptical and hostile attitudes on the part of (especially younger) group members towards autonomous driving could be down to the fact that life in the future was so clearly depicted as being full of structured and optimized everyday tasks.

On the other hand, the qualities and attributes ascribed to autonomous driving by the participants can clearly be classified in terms of the earlier surveys, with the same ascriptions cropping up again and again in both the exploratory study and the quantitative questionnaire.

\section{The "accelerated service society"}

That it will in future be possible to spend time on other activities in an autonomous vehicle was largely viewed negatively in the discussions. In their orally read-out introspection reports, the participants expressed concern about no longer needing to concentrate on the driving task in future. This, they thought, could lead to private, leisure, and work activities becoming too closely mixed up with one another. Ultimately, this line of thought runs, technology may further a trend that many people today already identify as alarming: a society ever-more oriented towards performance and efficiency:

Johanna $^{2}$ "And private life and working life are mixing more and more and you become a total workaholic."

Timo "This strain of having to do more and more things in the same place at the same time is increasing."

The freedom and the opportunity to occupy oneself during the car journey in other ways may result in pressure to put this time in the service of efficiency. Having to concentrate while driving conventionally, on the other hand, was painted in a positive light:

Johanna "That's actually a nice thing about driving, that you have to concentrate on it in the moment and you also do something with your hands, and you precisely aren't already checking emails from work. That starts when you're sat at your desk."

\footnotetext{
${ }^{2}$ The names of the participants have been changed to protect their anonymity, and the quotes have been translated.
} 


\section{"This dependency on technology"}

One further consequence linked to autonomous driving is a future dependency on technology, which may also entail an accompanying large degree of loss of control, which is viewed negatively. Technology dependence and loss of control are also seen as problematic because there is skepticism as to the reliability of a technology over whose decisions one will no longer have any influence:

Nico "In certain situations you can simply decide spontaneously, and you might actually have a much better feeling for it than the car."

Julian "And obviously it won't be possible to intervene immediately either if I first have to hear 5, 4, 3, 2, 1 ... beep."

Evidently, behind such worries lies a fundamental skepticism regarding technology, all the more so when it greatly impinges on individual safety, as in the case of autonomous driving:

Julian "Not even my kettle gets my blind trust, why should I then blindly trust my car with my life? I somehow find that incredibly disconcerting."

\section{Life is being "de-funned" and "you'll get idle"}

The abolition of the driving task in autonomous vehicles is viewed highly critically. The main idea here is that a vehicle in which one does not drive oneself would curtail fun, spontaneity, individuality, flexibility, and control (it is interesting to note here that the participants had actually been given the Full Automation with Driver for Extended Availability story, where - as is described in the scenario - the driver can certainly still drive themselves whenever they like):

Johanna "What is actually then the difference to public transportation? Because what I actually always appreciate about a car is that it is in my own hands-that I can judge for myself. And if I'm running a little late, then I can step on the gas a little."

The "de-funning" (Timo) comment refers on the one hand very specifically to losing the fun of driving, but also to the lifestyle that could have the fun removed from it by autonomous driving:

Bettina "You'll stop needing to move so much because you'll be able to get picked up by the car everywhere; you'll get lazy, you'll get idle, you'll only ever take the car, because it doesn't matter if you're feeling ill or how you are-you can take the car for every trip you make."

\section{Social isolation: "Nobody needs anybody anymore".}

The consequence of such "de-funning" and idleness is, in the view of the group members, that humans in the end will be replaced. "Nobody needs anybody anymore" (Inga), there 
would then be a machine for everything and, in this way, even thinking would be done for you:

Inga "The car drives, you no longer have to drive yourself. Food is delivered in one way or another, you cower a touch autistically in your apartment and grow dull, you don't have to think anymore, you google or subject yourself to cat videos. So you basically become completely stupid."

\section{System weaknesses: How truly "autonomous" are autonomous cars?}

Overall, trust in the safety of autonomous vehicles appears to still be on the low side. This leads the participants into wide-ranging speculation: "How predictable are these cars, then?" and, "What about when the system breaks down and there is no internet access?" (Nico), "Can they be driven remotely?" (Thorsten), "What happens when this system gets hacked?" and, "Can these autonomous cars really be autonomous?" (Bettina), "Just where, then, is the evidence that the whole thing is going to be safe?" (Herta).

Behind such questions and statements, there is great insecurity regarding the still-unknown technology, and the possibility that dangers may accompany it. At the same time, the participants currently appear to be completely unclear as to who is behind the development of this technology and who is responsible for the system's safety. Who would be liable in case of an accident? Who would accept the damages? And most important: Who would accept responsibility in the ethical sense?

\section{"Social and economic consequences"}

The examination of autonomous driving also turns up the worry that the technology will lead to job losses in various sectors (the automotive industry, taxi and delivery services, etc.). This is connected to the idea that autonomous vehicles will result in less variety across the spectrum of automobiles: "If you only have this large one" (Bettina), autonomous driving would in the end be accompanied by "greater monopolization" (Eddie).

\subsubsection{Summary}

The issues raised in the group discussions concerning autonomous driving are embedded in a sociotechnological context that simultaneously uncovers negative and problem-centered ideas of society in future. Overall, the participants tend to take a hostile position vis-à-vis autonomous driving. Although positive aspects are also perceived, these are neither embedded in a specific usage context nor associated with positive ideas of future societyat least not to the same extent as the negative ones.

Fears expressed in connection to a future with autonomous driving concern social isolation, social and economic consequences, over-reliance on technology, increasing idleness, and the pressure to keep up in a primarily performance-oriented society. 


\subsection{Conclusion}

The results from the quantitative questionnaire challenge the currently widespread assumption that autonomous driving can expect general openness and a high degree of acceptance (see [55-57]). Such openness is to be found in our study, but only when the question of what an autonomous vehicle is left unspecified. When the respondents are introduced to concrete use cases of autonomous driving, however, their assessments turn out to be far more negative. Assessments of Vehicle on Demand in particular suggest that some forms of autonomous driving are still relatively far away from people's specific ideas of what a vehicle is, and also their ideas of how to get around. Among other things, the negative assessment arises from people feeling more "exposed" to potential risks and dangers that may arise in a Vehicle on Demand than they feel able to "control", as they will no longer be able to control the vehicle themselves at all. Overall, it is currently debatable whether most people have any picture of what "autonomous driving" might mean. In the quantitative survey, $44 \%$ of respondents still indicated they knew nothing about the subject, which clearly shows that awareness of it is far from penetrating all corners of society. These findings may also be taken as a sign that further studies more closely focused on the uses and applications of autonomous driving are required.

Furthermore, the negative associations of autonomous driving and autonomous vehicles in particular indicate that a use-oriented examination must also address specific user groups. Scenarios to be worked with should, for instance, take even greater account of the different users' living environments and systems of relevance. In this connection, it is at least clear that the level of trust placed in the still relatively unknown technology is on the low side - it is doubted that autonomous vehicles can be safe at all. At the same time, people currently have neither any specific idea what the technology is capable of nor who is behind its development and, in case of doubt, would accept responsibility for any potential damage, for example.

The present remarks have primarily looked at people's "subjective understanding" and reflexive or theoretical knowledge ([58]: $10 \mathrm{ff}$., [54]: $40 \mathrm{ff}$.) of autonomous driving and car use in general. The aim here was to trace how those acting deal reflexively with the topic, and thus also trace their motives, and then to ask: How is car use understood from a subjective perspective? What do people think about while using one? What intentions inform their behavior? This level of meaning forms the "orientation scheme" of the action-guiding knowledge structure. In future empirical work, it will also be necessary to decipher the orientation frameworks, i.e. the structure of action, and to ask how the praxis of car usage is produced in milieu- and culture-specific terms.

Whether autonomous driving will provoke a fundamental transformation in the system of automobility can currently hardly be predicted - up to now there has been little sign that the hegemony of automobile usage is in serious danger. Nevertheless, in the end it may be precisely such a new technology as autonomous driving that sustainably and irreversibly transforms our transport system. As the sociologist John Urry puts it: "Just as 
the internet and the mobile phone came from 'nowhere', so the tipping point here will emerge unpredictably, probably from a set of technologies or firms or governments not currently a center of the mobility industry and culture" ([59]: 272).

Open Access This chapter is distributed under the terms of the Creative Commons Attribution 4.0 International License (http://creativecommons.org/licenses/by/4.0/), which permits use, duplication, adaptation, distribution and reproduction in any medium or format, as long as you give appropriate credit to the original author(s) and the source, a link is provided to the Creative Commons license and any changes made are indicated.

The images or other third party material in this chapter are included in the work's Creative Commons license, unless indicated otherwise in the credit line; if such material is not included in the work's Creative Commons license and the respective action is not permitted by statutory regulation, users will need to obtain permission from the license holder to duplicate, adapt or reproduce the material.

\section{References}

1. Anker, S.: Ist das die Zukunft unserer Mobilität? Die Welt. http://www.welt.de/debatte/ kommentare/article128675822/Ist-das-die-Zukunft-unserer-Mobilitaet.html (2014). Last accessed on 30 September 2014

2. Heise online: Autonome Autos machen Privat-Pkw überflüssig. http://www.heise.de/ newsticker/meldung/Autonome-Autos-machen-Privat-Pkw-ueberfluessig-1943508.html (2014). Last accessed on 30 September 2014

3. Der Stern/ DPA: So sollen Roboter-Autos den Verkehr revolutionieren. stern.de http://www. stern.de/auto/news/google-car-so-sollen-roboter-autos-den-verkehr-revolutionieren-2122143. html (2014). Last accessed on 30 September 2014

4. Hucko, M. Carsharing mit selbst fahrenden Autos: Daimler eifert Google nach. Spiegel Online http://www.spiegel.de/auto/aktuell/daimler-autobauer-plant-car-sharing-mit-autonomfahrenden-smarts-a-980962.html\#ref=rss (2014). Last accessed on 30 September 2014

5. Sheller, M.: The Emergence of New Cultures of Mobility: Stability, Openings, and Prospects. In: R. Kemp et al. (eds.) Automobility in transition? A socio-technical analysis of sustainable transport, pp. 180-202. Routledge, London (2011)

6. Gardner, B., Abraham, C.: What drives car use? A grounded theory analysis of commuters' reasons for driving. Transp. Res. Part F: Traff. Psychol. Behav.10, 187-200 (2007)

7. Ladd, B.: Autophobia. Love and Hate in the Automotive Age. The University of Chicago Press, Chicago (2008)

8. Miller, D.: Car Cultures. Berg, Oxford / New York (2001)

9. Curtis, C., Low, N.: Institutional Barriers to Sustainable Transport. Ashgate, Farnham (2012)

10. Sandoval. J.S.O., Cervero, R., Landis, J.: The transition from welfare-to-work: How cars and human capital facilitate employment for welfare recipients. Applied Geography 31, 352-362 (2011)

11. Urry, J.: The 'System' of Automobility. Theory, Culture \& Society 21, 25-39 (2004)

12. Geels, F.: The Dynamics of Transitions in Socio-technical Systems : A Multi-level Analysis of the Transition Pathway from Horse-drawn Carriages to Automobiles. Technology Analysis \& Strategic Management 17, 445-476 (2005) 
13. Sheller, M., Urry, J.: The City and the Car. International Journal of Urban and Regional Research 24, 737-757 (2000)

14. Stotz, G.: The Colonizing Vehicle. In: Miller, D.: Car Cultures, S. 223-244. Berg, Oxford / New York (2001)

15. Sheller, M.: Automotive Emotions: Feeling the Car. Theory, Culture \& Society 21, 221-242 (2004)

16. Dant, T.: The Driver-car. Theory, Culture \& Society 21, 61-79 (2004)

17. Thrift, N.: Driving in the city. Theory, Culture \& Society 21, 41-59 (2004)

18. Bergstad, C.J., Gamble, A., Hagman, O., Polk, M., Gärling, T., Olsson, L.E.: Affective-symbolic and instrumental-independence psychological motives mediating effects of socio-demographic variables on daily car use. Journal of Transport Geography 19, 33-38 (2011)

19. Williams, A.M., Shaw, G.: Future play: tourism, recreation and land use. Land Use Policy 265, 5326-5335 (2009)

20. Macintyre, S., Ellaway, A., Der, G., Ford, G., Hunt, K.: Do housing tenure and car access predict health because they are simply markers of income or self esteem? A Scottish study. Epidemiol Community Health 52, 657-664 (1998)

21. Brownstone, D., Small, K.A.: Valuing time and reliability: assessing the evidence from road pricing demonstrations. Transp. Res. Part A: Pol. Pract. 39, 279-293 (2005)

22. Bamberg, S., Ajzen, I., Schmidt, P.: Choice of travel mode in the theory of planned behaviour: the role of past behaviour, habit, and reasoned action. Basic and Applied Social Psychology 25, 175-188 (2003)

23. Li, Z.L., Hensher, D.A.: Congestion charging and car use: A review of stated preference and opinion studies and market monitoring evidence. Transport Policy 20, 47-61 (2012)

24. Litman, T.: Built for comfort, not just speed: valuing service quality impacts in transportation planning. Victoria Transport Policy Institute (2008)

25. Newman, P., Kenworthy, J.: Urban Design to Reduce Automobile Dependence. Opolis 2, 35-52 (2006)

26. Ewing, R.; Cevero, R.: Travel and the built environment. Journal of the American Planning Association 76, 265-294 (2001)

27. Mokhtarian, P. L., Salomon, L.: How derived is the demand for travel? Some conceptual and measurement considerations. Transport Research Part A 35, 695-719 (2001)

28. Steg, L., Vlek, C., Slotegraaf, G.: Instrumental-reasoned and symbolic-affective motives for using a motor car. Transportation Research Part F 4, 151-169 (2001)

29. Steg, L.: Car use: lust and must. Instrumental, symbolic and affective motives for car use. Transportation Research A 39, 147-162 (2005)

30. Gatersleben, B.: Psychological Motives for Car Use. In: T. Gärling et al. (eds.), Handbook of Sustainable Travel , S. 85-94. Springer, Dodrecht (2013)

31. Gatersleben, B., Uzell, D.: The journey to work: Exploring commuter mood among driver, cyclists, walkers and users of public transport. Environment and Behaviour 39, 416-431 (2007)

32. Ellaway, A., Macintyre, S., Hiscock, R., Kearns, A.: In the driving seat: psychological benefits from private motor vehicle transport compared to public transport. Transportation Research Part F 6, 217-231 (2003)

33. Lois, D, López-Sáez, M.: The relationship between instrumental, symbolic and affective factors as predictors of car use. Transportation Research Part A 43, 790-799 (2009)

34. Steg, L.; Vertoolen, L. G.J.: Affective motives for car use. In: European Transport Conference: Transport, Planning, Policy and Practice, Cambridge, London (1999)

35. Kent, J.: Secured by automobility: why does the private car continue to dominate transport practices? Dissertation, University of New South Wales, Australia (2013) 
36. Freudendahl-Pedersen, A.: Mobility in Daily Life - Between Freedom and Unfreedom. Ashgate, Farnham (2009)

37. Laurier, E., Lorimer, H., Brown, B., Jones, O., Juhlin, O., Noble, A., Perry, M., Pica, D., Sormani, P., Strebel, I., Swan, L., Taylor, A., Watts, L., Weilenmann, A.: Driving and 'passengering": Notes on the ordinary organization of car travel. Mobilities 3, 1-23 (2008)

38. Schuitema, G., Anable, J., Skippon, S., Kinnear, N.: The role of instrumental, hedonic and symbolic attributes in the intention to adopt electric vehicles. Transportation Research Part A 48, 39-49 (2013)

39. Freund, P., Martin, G.: The social and material culture of hyperautomobility: "Hyperauto". Bull. Sci. Technol. Soc. 29, 476-482 (2009)

40. Sheller, M., Urry, J.: The new mobilities paradigm. Environ. Plann. A 38, 207-226 (2006)

41. Gilroy, P.: Driving While Black. In: Miller, D.: Car Cultures, S. 81-104. Berg, Oxford / New York (2001)

42. Dowling. R.: Cultures of mothering and car use in suburban Sydney: a preliminary investigation. Geoforum 31, 435-353 (2000)

43. Augé, M.: Non-Places. Introduction to an Anthropology of Supermodernity. Verso, London (1995)

44. Baumann, Z.: 44 Letters from the Liquid Modern World. Polity Press, Cambridge (2010)

45. Laurier, E.: Doing office work on the motorway. Theory, Culture, Society 21, 261-277 (2004)

46. Laurier, E., Dant, T.: What We Do Whilst Driving: Towards the Driverless Car. In: Grieco, M., Urry, J.: Mobilities: New perspectives on transport and society, S. 223-243. Ashgate, New York / London (2012)

47. Mann, E. \& Abraham, C.: The role of affect in UK commuters' travel mode choices: An interpretative phenomenological analysis. British Journal of Psychology 97, 155-176 (2006)

48. Jain, J., Lyons, G.: The gift of travel time. Journal of Transport Geography 16, 81-89 (2008)

49. Jones, P., Thoreau, R., Massot, M.-H., Orfeuil, J.-P.: The Impact of Differences in Commuting Duration on Family Travel and Activity Patterns in the London and Paris Regions. In: Grieco, M., Urry, J.: Mobilities: New perspectives on transport and society, S. 179-205. Ashgate, New York / London (2012)

50. Kuhnimhof, T., Zumkeller, D., Chlond, B.: Who Made Peak Car, and How? A Breakdown of Trends over Four Decades in Four Countries. Transport Reviews 33, 325-342 (2013)

51. Kent, J.: Driving to save time or saving time to drive? The enduring appeal of the private car. Transp. Res. Part A. 65, 103-115 (2014)

52. Witt, H.: Introspektion. In: Mey, G., Mruck, K. Handbuch Qualitative Forschung in der Psychologie, S. 491-505. VS Verlag für Sozialwissenschaften, Wiesbaden (2010)

53. Kleining, G.: Lehrbuch Entdeckende Sozialforschung. Von der Hermeneutik zur qualitativen Heuristik. Psychologie Verlags Union, Weinheim (1995)

54. Bohnsack, R., Marotzki, W., Meuser, M. (eds.): Hauptbegriffe Qualitativer Sozialforschung. Barbara Budrich, Opladen \& Farmington Hills (2011)

55. Continental AG: Continental Mobilitätsstudie. http://www.continental-corporation.com/www/ download/presseportal_com_de/allgemein/automatisiertes_fahren_de/ov_mobilitaetsstudie_ 2013/download_channel/praes_mobilitaetsstudie_de.pdf (2013) Last accessed on 30 September 2014

56. AutoScout24 GmbH: Unser Auto von morgen 2013/14 http://ww2.autoscout24.de/special/ unserauto-von-morgen-2013-14/was-wuenschen-sichdie-europaeer-vom-auto-von-morgen/ 4319/392974/ (2013) Last accessed on 30 September 2014 
57. AutoScout $24 \mathrm{GmbH}$ : Unser Auto von morgen. Studie zu den Wünschen der Europäer an das Auto von morgen https://about.autoscout24.com/de-de/au-press/2012_as24_studie_auto_v_ morgen_en.pdf (2012) Last accessed on 30 September 2014

58. Bohnsack, R., Nentwig-Gesemann, I., Nohl, A.: Dokumentarische Methode und ihre Forschungspraxi. VS Verlag für Sozialwissenschaften, Wiesbaden (2013)

59. Urry, J.: Climate change, travel and complex futures. The British Journal of Sociology 59, 261-279 (2008) 